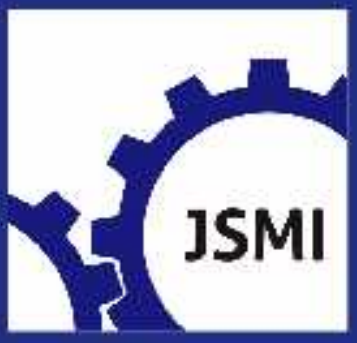

ISSN 2580-2887 (print)

ISSN 2580-2895 (online)

\title{
Jurnal Sistem dan Manajemen Industri
}

Vol 3, No 2, December 2019

A review of spare parts supply chain management

Zineb Achetoui, CharifMabrouki, Ahmed Mousrij

76.88

Performance measurement of public hospitals through the integration of SWOT and balanced scorecard

Yuli Setiawannie, Tiara Rahmania

Life cycle assessment analysis of kenaf cultivation in bonorowoland, laren, lamongan

Desrina Yusi Irawati, Lusi Mei Cahya Wulandari

Integrating e-servqual and kano model to improve adorable projects website service 98 - 106 quality

Rahma Fauziyah, Yati Rohayati, Boby Hera Sagita

Analysis and framework for agricultural supply chain improvement: a case study of 107 - 114 california papaya in cikarang

Johan K. Runtuk, Anstasia L. Maukar

A crow search algorithm for aircraft maintenance check problem and continuous airworthiness maintenance program

$115-123$

Nurhadi Siswanto, Asyraf Nur Adianto, Hasan Aji Prawira, Ahmad Rusdiansyah

Integrated analytic hierarchy process and mixed integer programming for supplier 124 - 133 selection in mold and dies industry

Agustinus Silalahi, Ronald Sukwadi, Duhita Al Hayyu Pramesjwari, Chendrasari

Wahyu Oktavia, Christine Natalia 


\title{
Jurnal Sistem dan Manajemen Industri
}

\author{
Vol 3, No. 2 , December 2019
}

\section{Editorial Arrangement}

\section{Editor in Chief}

Supriyadi, ST., MT., IPM. (Universitas Serang Raya, Indonesia)

\section{Editor Team}

Dr. Aries Susanty, ST, MT. (Universitas Diponegoro, Indonesia)

Firdanis Setyaning Handika, ST., MT. (Universitas Serang Raya, Indonesia)

Afni Khadijah ST., MT. (Universitas Banten Jaya, Indonesia)

Nurul Aziza, ST., MT. (Universitas Maarif Hasyim Latif Sidoarjo, Indonesia)

Eka Indah Yulistyari ST., MT. (Universitas Serang Raya, Indonesia)

Mohamad Jihan Shofa, ST., MT. (Universitas Serang Raya, Indonesia)

Nelfiyanti, ST. M.Eng. (Universitas Muhammadiyah Jakarta, Indonesia)

\section{Editorial Board}

Ir. Markus Hartono S.T., M.Sc., Ph.D., CHFP., IPM. (Universitas Surabaya, Indonesia) Professor António Abreu (Polytechnic Institute of Lisbon, Lisboa, Portugal) Željko Stević, Ph.D. (University of East Sarajevo, Bosnia and Herzegovina)

Erfan Babaee Tirkolaee. Ph.D. (Mazandaran University of Science and Technology, Iran) Magdy Helal, , Ph.D (American University of the Middle East, Kuwait)

Ronald Sukwadi, S.T., M.M., Ph.D. (Universitas Katolik Indonesia Atmajaya, Indonesia)

Yosef Daryanto, S.T.,MSc, Ph.D, IPM. (Universitas Atma Jaya Yogyakarta, Indonesia) Anak Agung Perwira Redi, Ph.D. (Universitas Pertamina, Indonesia)

L. Tri Wijaya Nata Kusuma, ST., MT, Ph.D. (Universitas Brawijaya, Indonesia)

Dr. Dani Yuniawan, ST., M.MT. (Universitas Merdeka Malang, Indonesia)

Dr.Iwan A Soenandi (Universitas Kristen Krida Wacana, Indonesia)

Dr. Novi Marlyana, ST., MT. (Universitas Sultan Agung Semarang, Indonesia)

Rahman Dwi Wahyudi S.T., M.T., MBA., IPM. (University of Surabaya, Indonesia)

Ing. Farid Wajdi, M.Sc. (Universitas Serang Raya, Indonesia)

Primahasmi Dalulia, S.T.,M.T. (Universitas Merdeka Malang, Indonesia)

\section{Editorial Address}

Main Building A, 2nd Floor

Institute for Research and Community Service

Universitas Serang Raya

Open Jurnal Sistem (OJS): http://e-jurnal.Ippmunsera.org/index.php/JSMI/index email jurnalsistemindustri@unsera.ac.id 


\section{TABLE OF CONTENT}

A review of spare parts supply chain management Zineb Achetoui, Charif Mabrouki, Ahmed Mousrij. $67-75$

Performance measurement of public hospitals through the integration of SWOT and balanced scorecard

Yuli Setiawannie, Tiara Rahmania $76-88$

Life cycle assessment analysis of kenaf cultivation in bonorowo land, laren, lamongan

Desrina Yusi Irawati, Lusi Mei Cahya Wulandari $89-97$

Integrating e-servqual and kano model to improve adorable projects website service quality

Rahma Fauziyah, Yati Rohayati, Boby Hera Sagita $98-106$

Analysis and framework for agricultural supply chain improvement: a case study of california papaya in cikarang

Johan K. Runtuk, Anstasia L. Maukar $107-114$

A crow search algorithm for aircraft maintenance check problem and continuous airworthiness maintenance program

Nurhadi Siswanto, Asyraf Nur Adianto, Hasan Aji Prawira, Ahmad Rusdiansyah $115-123$

Integrated analytic hierarchy process and mixed integer programming for supplier selection in mold and dies industry

Agustinus Silalahi, Ronald Sukwadi, Duhita Al Hayyu Pramesjwari, Chendrasari Wahyu Oktavia, Christine Natalia $124-133$ 